\title{
NEUROPSYCHOPHARMACOLOGY REVIEWS Sleep and neurological autoimmune diseases
}

\author{
Alex $\operatorname{Iranzo}^{1}$
}

Neurological autoimmune diseases are characterized by an inappropriate immune response that by mistake targets the nervous system. As a result, patients experiment a number of neurological manifestations that may include insomnia, excessive daytime sleepiness, cataplexy, central hypoventilation, and REM sleep behavior disorder. Polysomnographic evaluation may reveal disorganized sleep architecture involving both NREM and REM sleep, and REM sleep intrusions into wakefulness. The study of sleep disorders in the setting of autoimmune diseases (e.g., narcolepsy, anti-IgLON5 disease, paraneoplastic neurological syndromes) shows that an abnormal immune-mediated (humoral or cellular) response target the neuronal structures (e.g., brainstem, hypothalamus) and neurotransmitters systems (e.g., hypocretin) that regulate sleep resulting in sleep impairment. It is a window to examine the link between the autoimmune system and the sleep regulation at the molecular, cellular, and anatomic level.

Neuropsychopharmacology (2020) 45:129-140; https://doi.org/10.1038/s41386-019-0463-z

\section{INTRODUCTION}

An autoimmune disease is a condition characterized by an immune response that inappropriately targets own healthy organs. There are more than 70 autoimmune diseases including rheumatoid arthritis, psoriasis, multiple sclerosis (MS), type 1 diabetes, Hashimoto thyroiditis and Crohn's disease. The clinical symptomatology depends on which organs, tissues, and cells are affected. The neurological autoimmune disorders target structures of the central and peripheral nervous system and impair a number of neurotransmitter systems (Table 1 ). When the abnormal immune reaction attacks the areas of the central nervous system (CNS) that generate and modulate sleep the affected individual experiences sleep symptomatology. Symptoms include insomnia, excessive daytime sleepiness (EDS), circadian rhythm disorder, and dream-enacting behaviors resulting in REM sleep behavior disorder (RBD).

The association between autoimmune diseases and sleep symptomatology and sleep disorders is complex and multifactorial. Factors involved may be related to (1) a direct causal effect between autoimmune cellular or humoral mechanisms and sleep disorders/symptoms, (2) common risk factors between autoimmune diseases and sleep conditions, increasing their association, (3) typical symptoms of autoimmune diseases exerting an effect on sleep regulatory mechanisms (e.g., fatigue in MS), (4) effects of treatment (e.g., steroids inducing insomnia) other related medical factors (e.g., hospitalization in Guillain-Barré Syndrome), and (5) a coincidental association between autoimmune diseases and highly prevalent comorbid sleep disorders (e.g., insomnia).

This article reviews the sleep disorders that have an immunological pathogenesis (narcolepsy and anti-IgLON5 disease) and neuroimmunological disorders that show sleep abnormalities (MS, Guillain-Barré syndrome, and paraneoplastic neurological syndromes (PNS)). This review does not cover neurological and systemic autoimmune diseases where sleep abnormalities are not common such as neuromyelitis optica, myasthenia gravis, systemic lupus erithematosus, and Sjögren's syndrome.

\begin{abstract}
IMMUNOMEDIATED SLEEP DISORDERS
Narcolepsy with cataplexy

Definition. Narcolepsy with cataplexy (NC) (also known as narcolepsy type 1) is a chronic sleep disorder of autoimmune origin characterized by dysregulation of REM sleep secondary to impaired hypocretin (HCRT) neurotransmission in the brain. It is a neurological disease clinically characterized by severe and irresistible episodes of EDS, cataplexy (sudden onset of muscle paralysis triggered by strong emotions with retained consciousness), sleep paralysis, hypnagogic and hypnopompic hallucinations, automatic behaviors, fragmented nocturnal sleep, RBD, and weight gain. Car accidents and social, familial, professional, and psychological problems are common and can markedly impact the quality of life, particularly due to severe $\operatorname{EDS}[1,2]$.
\end{abstract}

Epidemiology. NC is a rare disease with a prevalence rate of $0.02-0.20 \%$ worldwide and $\sim 30$ cases per 100,000 inhabitants in Europe, Canada and the United States. Symptoms usually begin during adolescence or early adulthood but onset during childhood is not exceptional. NC affects men and women equally and symptoms persist throughout lifetime. More than $95 \%$ of the cases are sporadic but the disease has been described in some families and in monozygotic twins. The risk of a first-degree relative developing NC is $10-40$ times higher $(1-2 \%)$ than in the general population $[1,2]$.

Sleep symptomatology. NC is associated with abnormalities of REM sleep regulation consisting in complete or partial intrusions of this stage into wakefulness, resulting into episodes of irresistible sleepiness, cataplectic events, sleep paralysis, and hypnagogic hallucinations. In addition, patients with NC experience dreamenacting behaviors associated with increased muscle tone during

\footnotetext{
${ }^{1}$ Neurology Service, Sleep Center, Hospital Clinic de Barcelona, Barcelona, IDIBAPS, CIBERNED, Barcelona, Spain

Correspondence: Alex Iranzo (airanzo@clinic.cat)
}

Received: 3 March 2019 Revised: 17 June 2019 Accepted: 2 July 2019

Published online: 14 July 2019 
Table 1. Neuronal substrates, neuroanatomical areas, and main sleep symptomatology involved in the autoimmune conditions that are associated with sleep abnormalities

\begin{tabular}{|c|c|c|c|}
\hline & Neuronal substrate & Impaired regions & Sleep symptoms \\
\hline Anti-lgLON5 disease & Autoantibodies, Tau deposits & Hypothalamus, brainstem & $\begin{array}{l}\text { Hypersomnia, dream-enacting behaviors, } \\
\text { RBD, Stridor, Sleep apnea }\end{array}$ \\
\hline Kleine-Levin syndrome & Unknown & Thalamus & Episodes of hypersomnia \\
\hline Multiple sclerosis & Demyelinating plaques & Hypothalamus, brainstem & Insomnia, RBD, Secondary narcolepsy \\
\hline Guillain-Barré & $\begin{array}{l}\text { Demyelinating } \\
\text { polyradiculoneuritis }\end{array}$ & Unknown & Insomnia, vivid dreams \\
\hline LGI1 limbic encephalitis & Autoantibodies & Limbic system & Insomnia, hypersomnia, RBD \\
\hline Morvan's syndrome & Autoantibodies & Thalamus, limbic system & Insomnia \\
\hline $\begin{array}{l}\text { Paraneoplastic cerebellar } \\
\text { degeneration }\end{array}$ & Autoantibodies & Cerebellum & RBD \\
\hline
\end{tabular}

REM, a parasomnia termed RBD [1, 2]. Children with NC have a particular phenotype with movement disorders, early puberty, and weight gain [3].

The hypocretin system. The hypocretins (also known as orexins) are two peptides that were identified in the brain in 1998 [4]. HCRT1 (orexin-A) and HCRT2 (orexin-B) are produced exclusively by only 70,000 neurons located in the lateral and posterior hypothalamus. These two peptides are encoded by a precursor protein called pre-pro-hypocretin. HCRT1 and HCRT2 have respective receptors, named HCRT1 $R$ and HCRT2 R. HCRT1 has predominant affinity for HCRT1 R, whereas HCRT2 has similar affinity for both receptors (HCRT1 R and HCRT2 R). The HCRT neurons send and receive projections from and to multiple areas and nuclei in the brain that are involved in sleep regulation, including the locus coeruleus, locus subcoeruleus, dorsal raphe nucleus, tuberomammillary nucleus, basal forebrain, periaqueductal grey matter, ventral tegmental area, pedunculopontine nucleus, the entire hypothalamus, thalamus, amygdala, and cortex $[5,6]$. This indicates that the HCRT system interacts with the cholinergic, noradrenergic, dopaminergic, serotonergic, histaminergic, and GABAergic systems. HCRT is an excitatory neurotransmitter that promotes wakefulness and sleep-wake transitions, and inhibits REM sleep. The HCRT system is also involved in metabolic rate, feeding, locomotion, reward-seeking, affect, stress, motivation, and cognition [6].

Loss of the HCRT transmission is involved in NC in the same magnitude as insulin impairment is involved in type 1 diabetes. NC may occur in some particular breeds of dogs and a mutation in the gen that codes the HCRT2 receptors is responsible for the familial canine form of NC. One single human case with NC and undetectable HCRT1 in the CSF had a mutation in the HCRT gene [7]. The majority (90-95\%) of sporadic cases with NC has low or undetectable levels of HCRT1 in the cerebrospinal fluid (CSF) and no mutations in the HCRT genes and receptors have been identified in these sporadic cases [8, 9]. Postmortem examination of patients diagnosed with NC shows selective loss of $90-95 \%$ of the HCRT neurons in the posterior and lateral hypothalamus, whereas the nearby cells (e.g., the melanin-concentrating hormone neurons) are spared [5, 10]. In narcoleptic brains gliosis or inflammatory infiltrates are absent. This finding indicates that an insult destroyed only the HCRT neurons leaving intact the rest of the cells in the brain. The cause of the selective loss of the hypocretin-producing neurons in sporadic NC is still unknown.

The immunological basis. Although the cause of sporadic NC is uncertain, an autoimmune pathogenesis is strongly suspected. This is based on the following observations.

(1) Patients with NC have a genetic susceptibility to be affected by this disease. NC is tightly linked to the human leucocyte antigen (HLA) DQB1*0602 allele. About $95 \%$ of patients with NC are positive for this HLA subtype. This observation represents the strongest association of an HLA with any known disease. In contrast, the HLA variants $\mathrm{DQB} 1{ }^{*} 0601$ and $\mathrm{DQB} 1{ }^{*} 0603$ are protective for NC. The DQB $1{ }^{*} 0602$ allele is present in $\sim 25 \%$ of the Caucasian general population. Therefore, the absence of the HLA DQB1*0602 subtype argues against the diagnosis of NC, whereas its presence supports the diagnosis but is not a definitive feature since this is a relative common feature in the general population (25\%). Given that the prevalence rate of NC is only $0.02-0.20 \%$ in the general population, $>99 \%$ of HLA DQB $1{ }^{*} 0602$ positive individuals do not have narcolepsy. However, the HLA DQB $1{ }^{*} 0602$ allele increases the risk of developing NC 200-fold $[2,11]$. The tight link of NC with a HLA allele suggests that the disease is mediated by CD4+ T cells that might be implicated in the destruction of HCRT cells.

(2) An environmental factor (probably of microbiological origin) triggered an abnormal immunological response.

Streptococcal infection by the Streptococcus pyogenes is thought to be a trigger factor of the autoimmune process owing to the fact that increased anti-streptolysin $\mathrm{O}$ antibodies were found in some NC patients, particularly in those in whom the sample was taken close to NC onset [12].

In China, a marked increase of NC cases in children was temporally associated with the Influenza A H1N1 virus pandemia in 2010. All cases were DQB1*0602 positive [13].

An important number of new NC cases were linked to the H1N1 vaccination campaign that was carried in several European countries [14]. After the H1N1 seasonal vaccination campaign in Europe (between 2009 and 2011), an extraordinary increase of NC in children and adolescents was noted, particularly in those who were vaccinated with the AS03-adjuvanted of the Pandemrix vaccine. This excipient was associated with a 10-fold increase in new NC cases in children and adolescents and a four-fold increase in adults. Evaluation of the new affected cases showed that 
symptoms began few weeks after vaccination and demonstrated typical NC features such as HLA DQB $1{ }^{*} 0602$ subtype positivity and low levels of HCRT1 in the CSF. One study showed that the Pandemrix flu vaccine triggers antibodies that bind both the Influenza A virus and the HCRT2 receptor.

(3) There is an abnormal immunological response mediated by antibodies and cells that only destroyed the HCRT neurons in the hypothalamus $[15,16]$.

No specific autoantibodies have been identified in NC against HCRT or against HCRT receptors. However, some patients with NC have elevated autoantibodies against Tribbles homolog 2 (which is expressed by the HCRT neurons), particularly those with recent onset of symptoms. It is unknown if these antibodies are directly involved in the pathogenesis of the disease or are the result of the destruction of HCRT neurons.

Genome-wide association studies have shown association with polymorphisms in immune-mediated genes such as the T-cell receptor chain alpha gene locus (that in normal conditions recognize small peptides presented by the HLA system), purinergic receptor P2R11 gene, and variants in cathepsin $\mathrm{H}_{\text {, }}$ OX40L, CPT1B, and TNFSF4 genes.

Recent observations suggest that HCRT can be impaired by T cells, resulting in NC symptomatology. In patients with NC, circulating autoreactive CD4+ and CD8+ T lymphocytes are activated and react against to fragments of the pre-pro-hypocretin peptide precursor. This has been shown in adults and in children with sporadic NC, and in children in whom NC developed after influenza A H1N1 vaccination [15-17]. The important recent finding in narcoleptics that CD4+ and CD8+ autoreactive T cells target self-antigens of hypocretin-producing neurons highlighted that narcolepsy is an autoimmune mediated condition [17].

Taken together, it is thought that in genetically predisposed individuals carrying the $\mathrm{DQB} 1{ }^{*} 0602$ allele an environmental factor triggered an abnormal cellular and humoral immunological response. This response was mainly mediated by $\mathrm{CD} 4+$ and CD8 + T cells that can kill the HCRT neurons directly or indirectly activating the microglia and macrophages.

Diagnosis. Accurate diagnosis of the disease is crucial to (1) confirm the presence of a lifelong disorder linked to disability and socioeconomic difficulties, (2) start the available effective therapy (wake-promoting agents, sodium oxybate, antidepressants) in order to improve quality of life, (3) confirm the presence of a disease associated with a specific biological abnormality (deficient hypocretin transmission within the brain) that might be ideally treated with specific promising therapy (hypocretin replacement, hypocretin agonists, cell transplantation, gene therapy), when available, (4) exclude other causes of EDS and falls that may mimic the symptoms of narcolepsy (e.g., epilepsy, drop attacks, syncope, obstructive sleep apnea), and (5) identify malingering in people who feign symptoms to obtain psychoactive medications (e.g., stimulants, sodium oxybate) or social benefits (e.g., disability). The finding that narcolepsy is linked to sleep onset REM sleep periods (SOREMPs) has an important diagnostic value and also links with the pathophysiology of NC (loss of HCRT transmission). In 2014, the American Academy of Sleep Medicine published the third edition of the International Classification of Sleep Disorders where the diagnostic criteria of NC were established, and the disease was labeled Narcolepsy type 1 [1]. The diagnostic criteria for NC are the following and include clinical history, polysomnography followed by a multiple sleep latency test (MSLT) and assessment of HCRT1 levels in the CSF:

Criteria $A$ and $B$ must be met:

A. The patient has daily periods of irrepressible need to sleep or daytime lapses into sleep occurring for $\geq 3$ months.

B. The presence of one or both of the following:
1. Cataplexy and a mean sleep latency of $\leq 8 \mathrm{~min}$ and $\geq 2$ SOREMPs on an MSLT performed according to standard techniques. A SOREMP (within 15 min of sleep onset) on the preceding nocturnal polysomnography may replace one of the SOREMPs on the MSLT.

2. CSF HCRT1 concentration, measured by immunoreactivity, is either $\leq 110 \mathrm{pg} / \mathrm{mL}$ or $<1 / 3$ of mean values obtained in normal subjects with the same standardized assay.

Management. Currently, NC is an incurable disease because at the time of diagnosis most of the HCRT cells are lost, and HCRT peptide replacement and HCRT receptor agonists are not available. As the HCRT deficit is attributable to an autoimmune mechanism, immunosuppressive interventions (e.g., intravenous immunoglobulins, plasmapheresis, and corticosteroids) have been proposed but the reported response in a few cases has been only discrete, or no-beneficial therapeutic effect was noted.

The goal of therapy in NC is to allow the patient to have a full life and control the symptoms that are disabling and cumbersome [18-20]. Therapy should be tailored and comprises behavioral approaches and the use of drugs. Behavioral measures and sleep hygiene techniques should be recommended and consist in life counseling, scheduled short naps, regular sleep-wake habits, and avoid driving. Pharmacological treatments aim to control the narcoleptic symptoms. The medications that are used to treat EDS are CNS stimulants that promote adrenergic, noradrenergic, and dopaminergic activity. These wake-promoting agents are amphetamines, immediate and extended release formulations of methylphenidate, immediate and extended release formulations of modafinil, and solriamfetol. Most of the medications used to treat EDS have little effect on cataplexy and other REM sleepassociated phenomena such as sleep paralysis and hallucinations. Pitolisant improves EDS in NC owing to its histaminergic activity, and also improves cataplectic events. The medications used for cataplexy are tricyclics and selective serotonin reuptake inhibitors that promote noradrenergic and serotoninergic activity and in addition have an anticholinergic effect. They include clomipramine, venlafaxine, reboxetine, and fluoxetine, which are also effective for sleep paralysis and hypnagogic hallucinations, but have no effect on EDS. Sodium oxybate, a GABA-B receptor agonist, improve cataplexy, ameliorate sleep fragmentation, and has a moderate impact in EDS.

Future research and directions should involve the search for HCRT peptide replacement, HCRT receptor agonists, HCRT cell transplantation, gene replacement therapy, immunotherapy administered at disease onset, GABA-A receptor antagonists (clarithromycin, flumazenil), and melanin-concentrating hormone receptor antagonists.

Anti-lgLON5 disease

Definition. This is a novel neurological disease initially described in 2014 in eight unrelated individuals [21]. It is characterized by an heterogeneous neurological presentation, a distinct sleep pattern with abnormal sleep architecture, RBD, serum and CSF antibodies against the neuronal protein IgLON5, a strong HLA association, absence of coexistent autoimmune disorders, neoplasms, and neurodegenerative diseases, and a neuropathological pattern defined by tau deposits in the brainstem and hypothalamus impairing some nuclei that regulate sleep [21-48].

Clinical features. Mean age at diagnosis is $\sim 65$ years with an age range between 45 and 83 years. Clinical course at presentation is usually chronic (years) but the subacute form (months) is not rare. A few cases with acute onset (weeks) have also been described [36]. Median interval between symptom onset and diagnosis is 2.5 years (range, 2 months to 18 years) [30]. At referral, patients consult either to the general neurologist or to the sleep specialist, 
depending on the most predominant symptoms and severity. Reasons for referral are sleep symptoms, gait imbalance, dysphagia, and cognitive decline. At the initial visit, four main distinct clinical phenotypes, according to symptoms predominance, have been identified, but overlapping features among them are the rule. The four clinical presentations are the following: (1) a sleep disorder consisting in abnormal motor sleep behaviors, insomnia, EDS, sleep attacks, and sleep breathing symptoms, in any combination; (2) a bulbar syndrome that may include dysphagia, dysarthria, hypersalivation, and acute respiratory insufficiency that requires intubation and tracheotomy; (3) a progressive supranuclear palsy-like syndrome with abnormal gait, falls and gaze palsy, and (4) a Huntington disease-like syndrome with cognitive impairment and choreic movements of the limbs and face [30-44]. In addition, a motor neuron disease-like phenotype has been described in one patient from China with limb muscular weakness, dysphagia, and extensive denervation demonstrated by electromyography [37]. In some cases, presentation can be similar to some forms of subacute encephalitis characterized by confusion, somnolence, hallucinations, and fever [36-38]. Other signs and symptoms are cerebellar syndrome, a variety of oculomotor abnormalities, movement disorders, dysautonomic features, neuropsychiatric symptoms, fasciculations, muscle weakness, distal muscular atrophy, neck pain, frontal lobe seizures, and stiff-person like syndrome with cramps and limb stiffness. Fever may occur in cases with acute onset [36-38].

Sleep abnormalities. At the initial visit, about two thirds of the patients complained of sleep symptoms, namely continuous EDS, sleep attacks, insomnia-affecting sleep onset and sleep maintenance, witnessed apneic events, stridor, and abnormal sleep behaviors. Our impression, however, is that most patients suffer from sleep disorders. Sleep problems can be overlooked because other symptoms were prominent and patients and bed partners did not report sleep symptoms, and also because doctors did not ask about them. Direct questioning often reveals sleep symptoms. Most of the patients are unaware of their abnormal sleep behaviors that are only noted and reported by the bed partners. They include prominent jerks, vocalizations such as talking, and purposeful behaviors such as manipulating imaginary objects. Stridor and breathing pauses are reported only by the bed partners. Some patients may complain that they have prominent nocturnal enuresis. Patients have no previous history of disorders of arousal (sleepwalking, sleep terrors, confusional arousals) or sleep-related epilepsy.

Sleep architecture shows a very complex and novel pattern that for its identification needs full PSG with synchronized audiovisual recording and electromyographic leads in the four limbs [21] (Fig. 1). This V-PSG pattern is characterized by

1. Normal occipital alpha rhythm during wakefulness.

2. Mild reduction of total sleep time.

3. A distinctive temporal sequence of sleep abnormalities, from most abnormal at the beginning of the night to normalization at the end of the night.

4. Initiation of sleep and re-entering of sleep after awakenings characterized by theta activity with rapid repetitive and stereotyped leg movements separated by an interval of < 5 seconds that do not fit criteria for periodic leg movements in sleep.

5. N1 sleep and N2 sleep can be normal in some periods.

6. A novel sleep stage termed "undifferentiated NREM sleep" characterized by diffuse irregular theta EEG activity of 4-7 $\mathrm{Hz}$ with moderate amplitude (20-50 $\mu \mathrm{V})$, absence of vertex waves, $\mathrm{K}$ complexes, and sleep spindles, and usually associated with excessive electromyographic activation linked to frequent vocalizations, movements and complex behaviors.
7. Another novel stage termed "poorly structured N2 sleep" characterized by scant spindles and $\mathrm{K}$ complexes with frequent vocalizations (e.g., talking, laughing, crying), simple motor activity (e.g., raising the arm, punching), and finalistic behaviors (e.g., goal directed behaviors like sucking the thumbs while apparently eating, salting food, dabbing perfume, manipulating wires, picking up objects, knitting).

8. Normal N3 sleep that can be seen in the second half of the night.

9. RBD. REM sleep is characterized by increased electromyographic activity in the mentalis and in the four limbs. The most-frequent RBD manifestations are body and limb jerks. Aggressive behaviors such as punching and shouting in REM sleep occur only in few instances.

10. Obstructive sleep apnea with an apnea-hypopnea index ranging from 15 to 80 per hour associated with oxyhemoglobin desaturations.

11. Inspiratory stridor during sleep, particularly during normal $\mathrm{N} 2$ and N3 sleep.

In anti-IgLON5 disease, the sleep abnormalities challenge the identification of sleep and its stages. This indicates that scoring sleep in these patients is a difficult task because the usual parameters used to score sleep are often altered. The same scenario occurs in some subjects with neurodegenerative diseases, particularly when dementia is associated. In these instances, sleep scoring needs to be faced using a more descriptive and simple system with new rules rather than using the standard system that was originally delineated for nonneurologically impaired subjects.

It has to be noted that the most complex sleep behaviors are seen in non-REM sleep (and not in REM sleep) and they are different from the classical NREM parasomnias (sleepwalking, sleep terrors, confusional awakenings) since they are not abrupt and do not emerge from normal N3. Thus, anti-IgLON5 disease is not an overlap parasomnia.

The sleep pattern seen in the anti-IgLON5 disease cannot be considered status dissociatus because (1) wakefulness can be distinguished from sleep by clinical and polysomnographic features, (2) K complexes, spindles, and delta waves are present in NREM sleep, and (3) NREM sleep can be distinguished from REM sleep.

Agrypnia excitata is an abnormality that comprises familial fatal insomnia, Morvan's chorea and delirium tremens, which is characterized by insomnia, abnormal circadian pattern, disrupted sleep architecture, motor hyperactivity, confuse-oneiric state, prominent dysautonomia and thalamic damage. Anti-IgLON5 disease cannot be considered a form of agrypnia excitata because (1) there is no loss of sleep (total sleep time is mildly reduced but discernible), (2) K complexes, spindles, delta waves and REM sleep are always present and do not decrease with the progression of the disease, (3) episodes of REM sleep are not short, (4) circadian sleep-wake pattern is normal, (5) dysautonomia is not prominent, and (6) neuropathology shows that the thalamus is not damaged.

Immunological findings. Iglon5 is a normal cell adhesion protein located in the surface of the neurons. Its function is unknown. In the anti-lgLON5 disease autoantibodies against IgLON5 are always found in the serum and very frequently in the CSF. They represent the immune hallmark of the disease. IgG4 subclass antibodies predominate over IgG1 [30]. These IgLON5 antibodies are not found in idiopathic RBD, obstructive sleep apnea, neurological autoimmune disorders (e.g., MS) and neurodegenerative diseases (e.g., multiple system atrophy, Parkinson disease, progressive supranuclear palsy) $[21,45,46]$. Antibodies against IgLON5 have been found in one patient that fulfills clinical diagnostic criteria for progressive supranuclear palsy, but with an unusual clinical course of > 20 years [21]. Onconeuronal antibodies (e.g., LGI1, Caspr2, 


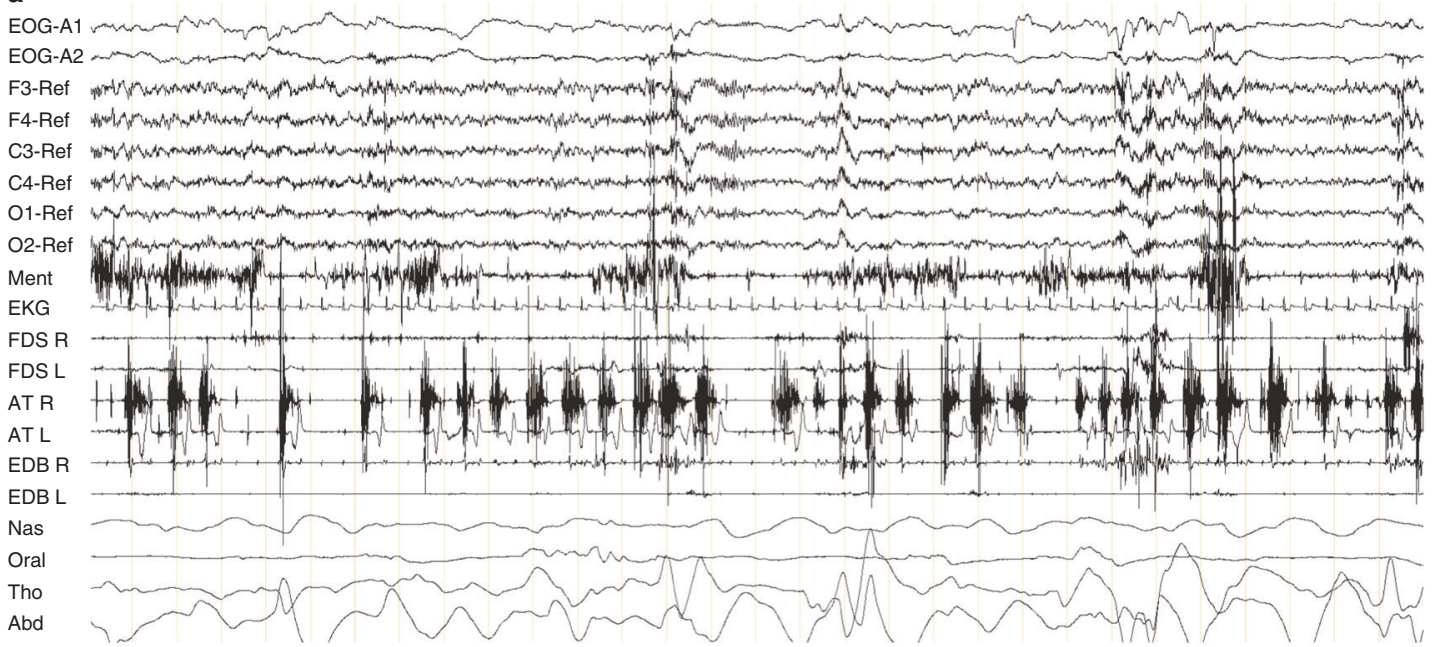

\section{b}
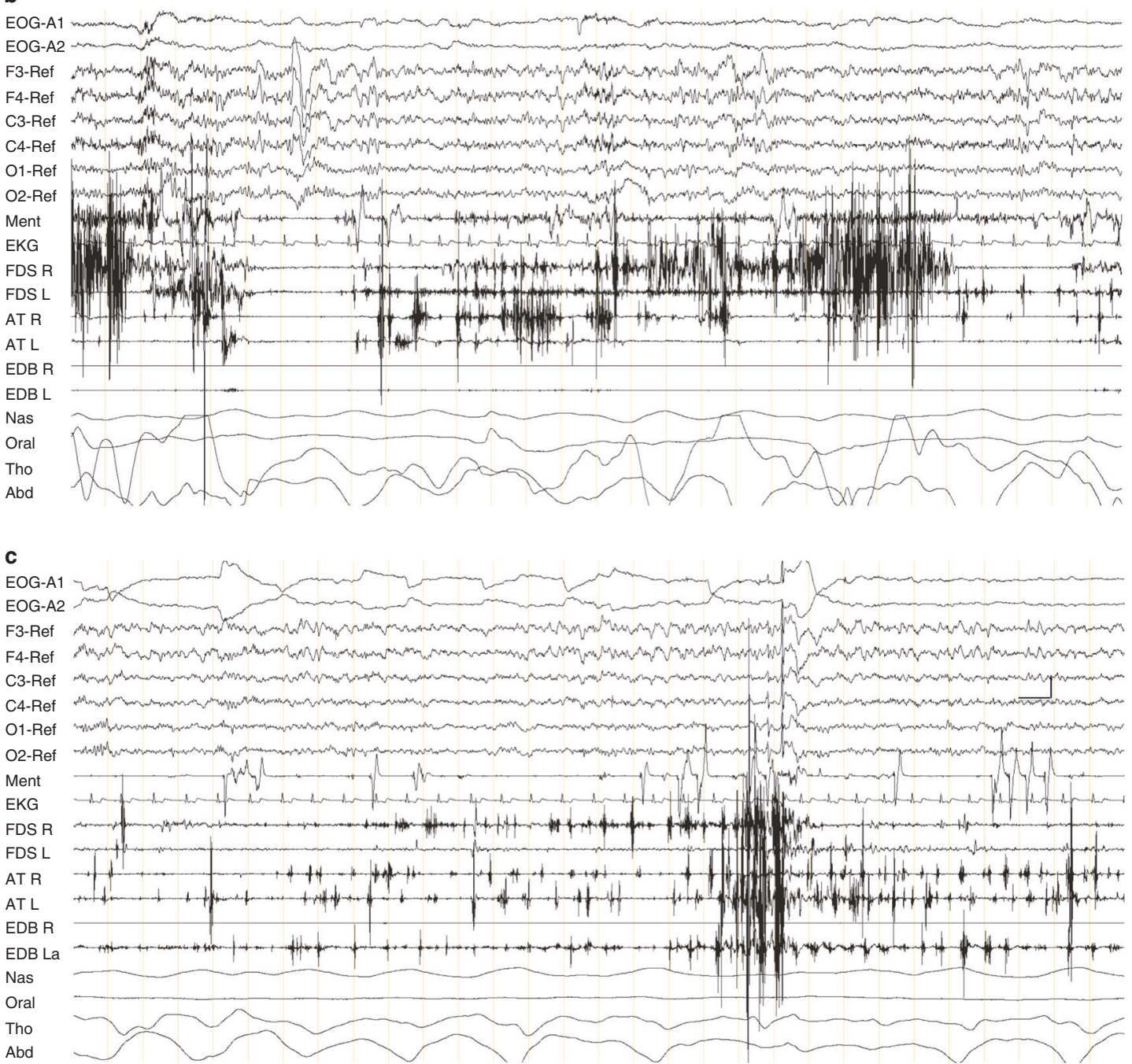

Fig. 1 Polysomnography findings from a patient with anti-IgLON5 disease showing "undifferentiated NREM sleep" a "poorly structured N2 sleep b, and "REM sleep behavior disorder" c. EMG: electromyogram; EOG: electrooculogram; Ment: electromyography of mentalis muscle; EKG: electrocardiogram; FDS: flexor digitorum superficialis muscle right (R) and left (L); EDB: extensor digitorum brevis muscle right (R) and left $(\mathrm{L})$; AT: anterior tibialis right (R) and left (L); NAS: nasal airflow; oral: oral airflow THO: thoracic respiratory movement; ABD: abdominal respiratory movement. Note the calibration mark for time/ EEG voltage c. All pictures represent a 30-second epoch. REF: EEG electrodes were referenced to both ears 
134

Ma2, Hu, amphyphisin, NMDA, AMPA, mGLuR1, mGluR5, DPX, $G_{B B A_{B}}$ ) are absent in patients with the anti-lgLON5 disease.

The haplotypes DRB1*1001 and DQB1 ${ }^{*} 0501$ are detected in $85 \%$ of the patients tested. They are uncommon in the general population. DRB1*1001 is 36 times more frequent in patients with anti-IgLON5 disease than in the general population. The $\mathrm{DQB} 1{ }^{*} 0501$ is 3.5 times more from frequent in the anti-lgLON5 disease than in the general population [30].

Clinical course and therapy. The introduction of anticholinergics such as clomipramine may worsen markedly the symptomatology. Vocal cord palsy and central hypoventilation are the causes of respiratory failure, a situation that requires intensive care support and tracheotomy. The prognosis of the disease seems to be poor in many cases. Immunotherapy (cycles of intravenous steroids, intravenous immunoglobulins, pulses of cyclophosphamide, rituximab, azathioprine, and plasmapheresis) are usually not helpful $[21,25,34]$. Some cases, however, have been described to improve after immunotherapy [27, 28, 32, 34, 36, 38, 39, 41, 43]. This is why immunotherapy has been proposed as first line therapy for anti-IgLON5 disease [43]. Most of the patients decease owing to sudden death both during wakefulness and sleep, and aspiration pneumonia.

Neuropathology. The initial description of the disease included the postmortem examination of two patients [21]. Neuropathology showed the absence of inflammatory infiltrates and the presence of neuronal loss, moderate gliosis, and extensive deposits of abnormal hyperphosphorylated tau (with the presence of three-repeat and four-repeat tau isoforms) mainly involving the neurons of the tegmentum of the brainstem and the hypothalamus. The glia is spared. Deposits of beta-amyloid and alphasynuclein are not seen. The nuclei damaged in the brainstem are the laterodorsal tegmental area, periaqueductal grey matter (which may explain the abnormal sleep pattern), pedunculopontine nucleus (that may cause disequilibrium with gait abnormalities and falls), and nucleus ambiguous (producing vocal cord palsy leading to stridor). The locus sobcoeruleus is preserved. Damage of the magnocellular nucleus in the medulla may explain the occurrence of RBD in view of the preservation of the locus subcoeruleus. Other structures affected are the hippocampus, hypothalamus, and amygdala. The cortex, thalamus, substantia nigra, basal ganglia, and cerebellum are preserved or mildly affected. The anatomical distribution of this taupathy in the brain is different from the primary taupathies (e.g., progressive supranuclear palsy, corticobasal syndrome).

Neuropathological criteria have been established for this new entity after the postmortem study of four additional cases [29]. Definite diagnosis of the anti-IgLON5 disease requires detection of serum or CSF IgLON5 antibodies plus neuronal loss, gliosis, and tau deposits in the neurons. Probable diagnosis is defined when the antibody status is unknown but there is compatible clinical picture, HLA DRB1*1001 and DQB1*0501 positivity and positive neuropathology. Possible diagnosis is considered in cases with compatible neuropathology but without information of the clinical features and immunological status (antibodies and HLA genotype). An additional postmortem study of a single case showed brainstem and hypothalamus tau deposits in addition to microglial and neuronal TDP-43 pathology in regions without tau involvement (e.g., thalamus and basal ganglia) [31].

In two living patients who underwent brain biopsy, the frontal cortex showed infiltrates of $\mathrm{T}$ lymphocytes, macrophages and glial cells, focal edema, and negative immunostaining for tau $[36,40]$.

The underlying substrate. In the anti-IgLON5 disease there is no evidence of malignancy. It is still unclear if we are facing a neurodegenerative or an autoimmune disease [47]. On one hand, some features suggest that the disease has an autoimmune origin (e.g., antibodies against a neuronal surface antigen, the fact that other antibodies against other members of IgLON protein family are involved in autoimmune diseases such as MS, and the strong HLA association). Alternatively, other findings suggest a neurodegenerative basis (e.g., no marked clinical improvement with immunosuppressive therapy, a chronic and progressive clinical course, and evidence of neuronal loss, tau deposits and absence of inflammatory infiltrates). The anti-IgLON5 disease suggests an intriguing link between autoantibodies and abnormal deposits of tau in the brain. An experimental study in rat hippocampus showed that IgLON5 antibodies recognized the antigen in the neuron surface within the neurons. Antibodies produce the internalization of the antigen, suggesting a pathogenic role of these antibodies [48]. It has been speculated that the antibodies interfere the interaction of IgLON5 protein with the internal cytoskeletal network, leading to abnormal tau accumulation and ultimately neuronal loss [30]. Further studies are needed to clarify the origin and pathogenesis of the disease.

Kleine-Levin syndrome. Kleine-Levin syndrome is characterized by recurrent episodes of EDS, abnormal cognition, eating abnormalities and sexual disinhibition. The pathophysiology of KLS remains unknown [1]. An autoimmune basis has been thought because it is relapsing-remitting, can be triggered by infections and one study linked the condition with the DRB $1{ }^{*} 0301-\mathrm{DQB} 1{ }^{*} 0201$ and DRB1*0701-DQB1*0202 haplotypes [49].

\section{SLEEP ABNORMALITIES IN NEUROIM1MUNOLOGICAL DISEASES}

Multiple sclerosis

Definition. MS is a common chronic inflammatory demyelinating disease of the CNS. MS affects multiple regions of the CNS resulting in a wide variety of neurological manifestations. The cause of MS is unknown, but an abnormal immune-mediated response against antigens expressed in the cells of the CNS is strongly believed to play a crucial role. Genetic and environmental factors are also involved. MS is presumed to be a consequence of infiltrating $T$ lymphocytes that are reactive to myelin proteins and induce an inflammatory response that destroys the myelin and the axons. The pathology of MS consists in demyelination, axonal loss, inflammation, and gliosis.

MS is often seen in young people with usual onset between the ages of 20 and 45 years, with clinical relapsing-remitting and chronic progressive forms. An important variety of symptoms and signs (e.g., motor, sensitive, visual, autonomic) affecting different regions of the brain and spinal cord are characteristic.

A variety of sleep disorders can also be seen in patients with MS, impairing the quality of life. In MS, sleep disorders are more common than in the general population and the prevalence is $40 \%$. In general, sleep disorders in MS are more common in women than in men. Multiple factors contribute to the occurrence of sleep disorders in MS including depression, anxiety, fatigue, the effect of some medications, inflammation, and localization in the brain of the demyelinating lesions [50-52].

Insomnia. Insomnia occurs in $\sim 40 \%$ of patients with MS and includes sleep onset insomnia, nocturnal fragmentation owing to frequent and long awakenings during the night, and early awakenings. In some cases, insomnia may be linked to a circadian rhythm disorder. It seems that insomnia is more common in MS patients with older age, longer duration of the disease, progressive primary forms and greater disability. The causes of insomnia in MS are multiple and include anxiety, depression, restless legs syndrome (RLS), nocturia, spasticity, pain, and the use of some medications such as interferon and corticosteroids [50-53]. 
$R L S$. RLS-like symptoms are reported in up to $20 \%$ of the patients with MS, a prevalence rate that is higher than in the general population (5\%) [50-53]. Clinical onset of RLS usually follows the typical MS symptomatology. However, some studies have not shown that RLS is more common in MS than in controls [54]. It is possible that some MS symptoms (spasticity, paresthesia, cramps, pain and numbness) may be the source of false positives for RLS (the RLS mimics). The link between MS and RLS, if it does exist, is unclear, with only a few cases associated with impairment of iron metabolism and spinal cord demyelinating lesions in the cervical region.

RBD. RBD has been described in a few patients with MS, particularly when the demyelinating plaque is located in the pontine tegmentum, presumably impairing the nuclei and pathways that regulate REM sleep muscle atonia.

In a study comprising 135 consecutive MS patients, the individuals were interviewed for symptoms suggestive of RBD using a semi-structured questionnaire [54]. Four of the 135 patients reported symptoms suggestive of RBD that were later re-evaluated by a sleep disorders specialist. V-PSG confirmed RBD in three of these four patients, giving an estimated frequency of RBD of $1.4 \%$. None of these three patients had previously consulted to their doctors because of RBDrelated symptoms. Two of the three RBD patients were taking antidepressants, and RBD onset coincided with the introduction of the drug in one of these two. Because the antidepressant drug of this patient could not be withdrawn owing to severe depression, the cause of RBD was thought to be linked to the antidepressant intake. In the second patient, RBD onset was not related to a previous relapse, and a second V-PSG study confirmed the presence of RBD after withdrawing the drug for 1 month. The third patient was the only patient in whom brain magnetic resonance imaging showed a demyelinating plaque in the dorsal pons. Treatment with clonazepam at bedtime completely resolved the RBD symptoms in this third patient.

RBD can rarely occur as the presenting symptom of MS. RBD was reported as the initial manifestation in a 25 -year-old woman with a 6-month history of dream-enacting behaviors. She had sudden awakenings from fearful dreams with screams kicking, running to the door or to the window, crying, and falling out of bed resulting in injuries. If awakened, she always recalled a fighting dream. RBD was confirmed by V-PSG and brain imaging disclosed multiple cerebral periventricular and pontine demyelinating plaques consistent with a diagnosis of probable MS. RBD episodes disappeared after immunotherapy [55].

A 38-year-old woman had 1-year history of dream-enacting behaviors and nightmares. Polysomnography demonstrated RBD showing increased phasic electromyographic activity in the four limbs during REM sleep. Neurological examination was normal. Brain magnetic resonance imaging showed multiple demyelinating lesions in the hemispheres, corpus callosum, periventricular regions, cerebellar peduncles, and dorsal pons. The CSF contained oligoclonal bands and therefore the diagnosis of MS was made. RBD symptoms ameliorated with clonazepam and fingolimod. The authors speculated that the dorsal pontine plaque caused RBD [56].

In another case report, a 51-year-old woman with MS developed acute vertigo, ataxia, diplopia, dysarthria, and bifacial weakness. Her husband described that she exhibited nightly sleep-related groaning, screaming, limb jerking, flailing, and violent thrashing. She did not recall these events or unpleasant dreams. Brain magnetic resonance imaging showed a large confluent area of increased T2 signal in the dorsal pons. V-PSG showed excessive tonic and phasic muscle activity in the chin, arms, and legs electromyographic leads during REM sleep, which was associated with vocalizations, arms and legs jerking.
Clonazepam resulted in significant improvement in the frequency and severity of RBD symptoms [57].

Secondary narcolepsy. In MS, narcolepsy-like cases occur when the demyelinating plaques damage the hypothalamus bilaterally resulting in HCRT deficit. The HCRT1 levels in the CSF are normal (both during attacks and during remission) in MS patients with the relapsing-remitting form in whom brain magnetic resonance imaging excluded the occurrence of demyelinating lesions in the hypothalamus [52].

Sleep disordered breathing disorders. Lesions in the pontine tegmentum and dorsal medulla have been linked to obstructive and central sleep apnea [52].

\section{Guillain-Barré syndrome}

Definition. Guillain-Barré syndrome (GBS) is an acute autoimmune demyelinating polyradiculoneuritis causing peripheral paralysis. CNS impairment may occur in few cases during the acute phase of the attack consisting in psychosis and mental confusion. In these cases with CNS impairment sleep disorders may occur. HCRT1 levels in the CSF may be undetectable in patients with features indicating CNS dysfunction (e.g., respiratory failure, dysphagia, dysarthria), particularly early in the disease course [58].

Sleep abnormalities. During the acute phase, at the time of hospital admission, patients commonly report poor sleep quality, sleep onset insomnia, fragmented nocturnal sleep, early awakenings, and reduced nocturnal sleep time. These complaints are associated with anxiety, pain, paresthesia, and motor impairment $[59,60]$. Polysomnographic studies performed at admission in the acute phase reveals reduced total sleep time and sleep efficiency, decreased REM sleep time, and both increased wake time after sleep onset and periodic limb movement index. The apnea-hypopnea index is high in patients with cranial nerve impairment and bulbar symptoms. The day after polysomnography, the mean sleep latency test shows normal mean sleep latency $(11.8 \mathrm{~min})$ without SOREMPs. Re-evaluation of the patients 1 month after immunotherapy (when patients show good recovery of their sensori-motor manifestations) shows the same polysomnographic abnormalities found in the acute phase, and similar mean sleep latency in the MSLT [61].

The scenario is different in patients with severe GBS that need to be admitted in the intensive care unit. In one study, 13 patients admitted in the intensive care unit were evaluated with a portable polysomnographic montage including mental electromyography (but not electromyographic leads in the limbs or video recording) [62]. Patients were evaluated during the acute attack, and seven had abnormal mental status and six normal mental status. HCRT1 levels in the CSF were normal. In patients who had visual hypnagogic hallucinations and vivid dreams, polysomnographic recordings showed disorganized sleep pattern in the patients with abnormal mental status characterized by abnormal sleep architecture (frequent shifts between sleep, NREM sleep and REM sleep, rapid eye movements in NREM sleep, short REM sleep latency, and REM sleep with atonia).

Paraneoplastic syndromes

PNS are uncommon neurological disorders related to neoplasms outside the CNS such as limbic encephalitis and subacute cerebellar syndrome $[63,64]$. PNS are not caused by metastases or infiltration of a tumor in the brain. PNS are immune-mediated disorders linked to onconeuronal antibodies against neural antigens expressed by both the tumor and the nervous system. Antibodies react with an antigen located both in the tumoral cells and cells of the nervous system, usually neurons and Purkinje cells. The immune system recognizes a protein expressed by the tumor 
136

as foreign and attacks this protein in the normal brain. Antibodies react with specific proteins expressed in the cytoplasm, nuclei, or surface membrane. In most PNS, the direct pathogenic role of the antibodies is debatable. Autopsy usually shows neuronal loss, gliosis, and inflammatory infiltrates of cytotoxic $T$ lymphocytes. Interestingly, PNS may precede the diagnosis of the underlying systemic cancer. PNS are common in patients with lung, ovary, breast, testicular cancer, and Hodgkin's disease. The clinical course is usually subacute and progressive. Symptomatology can be severe and involve any area of the nervous system. Neurological symptomatology depends on the brain structures where antigens are prominently expressed in the brain. Damage of dorsal root ganglia, cerebellum, amygdala, hippocampus, brainstem, hypothalamus, and thalamus are common in PNS. Neurological symptoms include confusional state, cognitive impairment, memory loss, seizures, movement disorders, psychosis, and sleep disorders $[63,64]$

This review covers the sleep problems that can be found in the PNS anti-Ma2 encephalitis, Morvan's syndrome, LGI1 limbic encephalitis, paraneoplastic cerebellar degeneration and antiNMDA encephalitis. It should be noted that anti-NMDA encephalitis and LGI1 limbic encephalitis are not always associated with neoplasms.

Sleep disorders in patients with PNS have received attention only recently [65]. Prospective and well-designed studies have not been published. Only small series and case reports have described patients with PNS suffering from sleep disturbances such as insomnia, EDS, RBD, and central respiratory abnormalities. In most of the reported cases sleep studies were not performed and CSF HCRT1 was not measured. Severity of sleep problems in patients with PNS ranges from mild to severe. Sleep disorders are not the only manifestation of the PNS as they coexist with limbic syndrome, brainstem symptomatology, and hypothalamic disturbances. Rarely, sleep abnormalities are the first or most-severe complaint among patients with PNS. PNS patients with sleep problems usually have coexistent damage of the brainstem, limbic system, and diencephalic structures including the hypothalamus. Hypersomnia has frequently described in patients with anti-Ma2 encephalitis, a PNS affecting diencephalic, limbic, and brainstem structures in any combination. Central hypoventilation may be seen in patients with (1) anti-Hu brainstem encephalitis associated with small cell lung cancer and (2) anti-NMDA receptor encephalitis linked to ovarian teratoma. Morvan's syndrome associated with malignant thymoma results in insomnia. Limbic encephalitis related to voltage-gated potassium channel complex anti-LGI1 may be associated with insomnia, hypersomnia, and RBD. In contrast, sleep problems seem to be uncommon in PNS patients with (1) sensory neuronopathy owing to anti-Hu antibodies against the dorsal root ganglia and (2) subacute cerebellar syndrome associated with anti-Yo antibodies against Purkinje cells. However, it should be noted that it is possible that the frequency and nature of sleep disorders in the setting of any PNS may have been overlooked because physicians frequently pay little attention to sleep medicine and because patients and bed partners usually do not spontaneously report them.

Anti-Ma2 encephalitis. Anti-Ma2 encephalitis is a PNS affecting diencephalic, limbic, and brainstem structures in any combination that it is usually linked to lung and testicular cancers $[63,64]$.

EDS: Most reported PNS cases with EDS had anti-Ma2 antibodies linked to lung and testicular cancers, probably because this PNS commonly affects diencephalic structures. In a detailed review of 38 cases with anti-Ma2-associated encephalitis the authors noted that "EDS affected $32 \%$ of the patients, sometimes with narcolepsy-cataplexy and low CSF HCRT" [66]. Of the 13 patients that developed hypothalamic dysfunction, 12 had EDS and two of them presented a narcoleptic phenotype characterized by cataplexy and hypnagogic hallucinations. One of the patients without cataplexy was "lethargic and napping more than usual" and CSF HCRT was undetectable, whereas magnetic resonance imaging showed abnormalities in the right thalamus, midbrain, and mesial temporal lobe but not in the hypothalamus [67]. This review [66] included six patients with anti-Ma2 encephalitis previously published [68] in whom CSF HCRT was undetectable in four patients with EDS and was normal in two patients without EDS. None of these six patients underwent polysomnographic sleep studies and the presence of cataplexy could not be determined because the study was retrospective. In another case series that involved 22 newly diagnosed patients with anti-Ma2 encephalitis four presented with EDS but sleep studies and HCRT status were not evaluated [69].

In addition, some case reports have described subjects with EDS and paraneoplastic anti-Ma2 encephalitis. One patient with testicular carcinoma presented with a full-blown limbic encephalitis syndrome plus EDS and brief cataplectic episodes triggered by emotions. Nocturnal polysomnography showed reduced sleep efficiency of $44 \%$ and a reduced sleep latency onset of $4.5 \mathrm{~min}$. A MSLT performed the following day demonstrated a sleep latency of $9 \mathrm{~min}$ with two SOREMPs. HLA DR2 was negative. Magnetic resonance imaging revealed bilateral medial temporal lobe damage but no hypothalamic impairment [70]. In another case report, a patient with anti-Ma2 encephalitis presented with subacute onset of severe hypersomnia, memory loss, parkinsonism, and gaze palsy. Brain magnetic resonance imaging showed bilateral damage in the dorsolateral midbrain, amygdala, and paramedian thalami. Video-polysomnography disclosed RBD, a reduced sleep efficiency of $48 \%$ and the MSLT showed a mean sleep latency of $7 \mathrm{~min}$ and four SOREMPs. HLA typing was negative for the $\mathrm{DQB} 1{ }^{*} 0602$ and $\mathrm{DRB} 1{ }^{*} 15$ antigens. CSF HCRT level was low (49 pg/mL) [71]. A patient with anti-Ma2 encephalitis and lung cancer presented with EDS, absence of cataplexy and hallucinations, and low CSF HCRT level $(58 \mathrm{pg} / \mathrm{ml})$. Magnetic resonance imaging showed prominent high signal intensity in the hypothalamus and hippocampus [72]. Hypothalamic damage demonstrated by magnetic resonance imaging was documented in another patient with anti-Ma2 encephalitis and breast cancer who presented with severe hypersomnia. Sleep studies, HLA typing and HCRT measurement were not assessed [73]. Other case reports noted the presence $[74,75]$ or absence [76-78] of EDS in subjects with anti-Ma2-associated encephalitis. A 55-year-old man presented with hypersomnolence, cataplexy, abnormal sleep behaviors, parkinsonism, and vertical supranuclear palsy. Polysomnography showed disrupted sleep architecture, RBD, and MSLT demonstrated reduced sleep latency and multiple SOREMPs. The patient had positive Ma1 and Ma2 antibodies and a tonsillar squamous cell carcinoma [79]. A 63-year-old man with anti-Ma2 antibodies had diencephalic encephalitis with EDS, cataplexy, HCRT deficiency, and RBD demonstrated by polysomnography. MSLT showed two SOREMPs and a mean sleep latency of 7 min Neuropathology demonstrated inflammation induced by cytotoxic CD8+ T lymphocytes and complete loss of HCRTergic neurons within the hypothalamus [80].

RBD: There are a few descriptions of RBD associated with antiMa2 encephalitis. In these cases, RBD seems to be linked to secondary narcolepsy owing to an inflammatory process involving the hypothalamus, amygdala, and brainstem. RBD, in these cases, is not the sole manifestation of the anti-Ma2 encephalitis where it is associated with coexistent cognitive impairment, gaze palsy, hypersomnia, and cataplexy. In anti-Ma2 encephalitis, RBD is usually mild and not a prominent feature of the clinical picture.

A 69-year-old man with anti-Ma2 encephalitis presented with subacute onset of severe hypersomnia, memory loss, parkinsonism, and gaze palsy. Brain magnetic resonance imaging showed bilateral damage in the dorsolateral midbrain, amygdala, and 
paramedian thalami. V-PSG disclosed RBD, reduced sleep efficiency of $48 \%$, and absence of sleep spindles. REM sleep was characterized by increased phasic and tonic muscular activity in the submentalis muscle and four limbs associated with kicking and prominent limb and trunk jerking. The MSLT showed a mean sleep latency of $7 \mathrm{~min}$ and four SOREMPs. HLA typing was negative for the $\mathrm{DQB} 1{ }^{*} 0602$ and $\mathrm{DRB} 1{ }^{*} 15$ alleles. CSF hypocretin level was low $(49 \mathrm{pg} / \mathrm{mL})$ [71].

A 55-year-old man presented with hypersomnolence, cataplexy, abnormal sleep behaviors, parkinsonism, and vertical supranuclear palsy. Polysomnography showed disrupted sleep architecture and complete loss of REM sleep atonia. During REM sleep, vocalizations (talking, laughing, and singing) and arms and legs movements were observed. MSLT demonstrated reduced sleep latency of 2 min and multiple SOREMPs without muscle atonia. The patient had positive Ma1 and Ma2 antibodies and a tonsillar squamous cell carcinoma was discovered [79].

A 63-year-old man with anti-Ma2 antibodies had diencephalic encephalitis with EDS, cataplexy, and HCRT deficiency. Polysomnography demonstrated fragmented and reduced sleep efficiency and sustained muscle activity in REM sleep. Neuropathology demonstrated inflammation induced by cytotoxic CD8+ T lymphocytes and complete loss of HCRT neurons within the hypothalamus [80].

A similar case report described a 67-year-old man with anti Ma1 and Ma2 antibodies admitted with a one-month history of EDS, confusion, memory loss, hallucinations, disorientation, hypophonia, and falls. Brain magnetic resonance imaging demonstrated a lesion in the hypothalamus. Right hypothalamic biopsy revealed lymphocytic infiltrate. A daytime nap of 128 min showed RBD [81].

Limbic encephalitis associated with LGI1 antibodies (previously termed potassium channel antibody-associated limbic encephalitis). The antibodies are active against the leucine-rich glioma inactivated protein that is part of the voltage-gated potassium channel complex, and is located in the surface of the neuron. This limbic encephalitis affects middle age or elderly individuals, mainly men. It is usually benign but in $10 \%$ of the cases it is associated with thymomas. In this condition there is a primary insult in the mesial temporal lobe involving the hippocampus and the amygdala, with no apparent direct involvement of the brainstem. Patients present subacute progressive cognitive impairment, confusion, disorientation in time and space, memory loss, hyponatremia, and seizures including piloerection [63, 82].

Sleep disorders such as insomnia, EDS, and RBD have also been described in a few subjects with limbic encephalitis associated with LGI1 antibodies. The clinical syndrome is partially or totally reversible with immunotherapy. The association of RBD with this limbic encephalitis served to establish a pathophysiological link between this parasomnia and impairment of the limbic system, which could explain the intense emotions occurring in the RBDrelated dreams (e.g., being attacked and chased). Other antibodies against the potassium channel complex bind the contactinassociated protein-2 (Caspr2) and the resulting neurological disorder consists in Morvan's syndrome, encephalitis, and neuromyotonia (a form of peripheral nerve hyperexcitability clinically characterized by slow muscle relaxation, muscle cramps, and stiffness). The identity of other potassium channel complex antibodies is unknown.

In one study, of six patients with non-paraneoplastic limbic encephalitis associated with LGI1 antibodies, five had the typical clinical history of RBD in the context of encephalopathy, seizures, and EDS. HCRT levels in the CSF were normal. Videopolysomnography could be performed in three of these five patients and demonstrated RBD showing increased electromyographic activity in REM sleep linked to prominent limb jerking. In three patients, immunosuppression resulted in resolution of RBD in parallel with remission of the limbic syndrome and disappearance of mesial lobe hyperintensity. RBD persisted in two patients with partial resolution of the limbic syndrome [83].

In another study, 15 patients were identified with antibodies against to voltage-gated potassium channels (specific LGI1 and Caspr2 antibodies were not analyzed in this study) and had limbic encephalitis $(n=5)$, Morvan's syndrome $(n=4)$, and overlapping features $(n=6)$. Clinical history revealed that two patients had hypersomnia, 10 prominent insomnia, and 8 dream-enacting behaviors (four with Morvan's syndrome, three with limbic encephalitis, and one with overlapping features). Polysomnography in seven of the eight patients with dream-enacting behaviors showed normal REM sleep in one, absence of REM sleep in three, and REM sleep without atonia (but without abnormal behaviors in REM sleep) in three (one with Morvan's syndrome, one with limbic encephalitis, and one with overlapping features) patients [84].

A 62-year-old man with limbic encephalitis associated with LGI1 antibodies had RBD since V-PSG showed that REM sleep contained increased phasic electromyographic activity associated with multiple kicks and jerks [85].

Morvan's syndrome (also known as Morvan's fibrillary chorea and Morvan's chorea). Morvan's syndrome is characterized by subacute onset of severe insomnia (usually not associated with hypersomnia), disrupted sleep-wake pattern, abnormal nearly continuous motor activation, mental confusion, visual daytime hallucinations, dysautonomia (constipation, salivation, lacrimation, urinary incontinence, hyperhidrosis, increased body temperature, tachycardia, hypertension) fasciculations, myoclonic jerks, cramps, and neuromyotonia. It is associated with Caspr2 antibodies in the context of myasthenia gravis, malignant thymoma, or small cell lung cancer. In most cases, the syndrome responds to immunotherapy but some cases worsen till death [86].

The sleep abnormalities that characterize Morvan's syndrome are insomnia and an extreme expression of state dissociatus due to breakdown of the sleep-wake boundaries [87-91]. This state is termed agrypnia excitata, which also is present in fatal familial insomnia and alcohol withdrawal syndrome (delirium tremens) [91]. It is thought that agrypnia excitata represents a thalamolimbic system dysfunction, although brain imaging is normal in Morvan's syndrome. In agrypnia excitata, the patients present severe insomnia, generalized sympathetic dysautonomia and nocturnal motor over-activation where subjects perform simple or complex behaviors mimicking daily-life activities such as eating, setting up a device, buttoning the pyjamas, pointing something in the wall or dressing. This nocturnal finalistic activity is labelled oneiric stupor, and is nearly continuous during the entire night with quiet pauses of several minutes. Episodes of oneiric stupor can occur with open or closed eyes. If questioned during one of these episodes, patients may respond that they are awake, although they seem to be behaving in the context of a dream or a hallucination. The oneiric stupor episodes can emerge from any stage (relaxed wakefulness, NRMEM sleep, and REM sleep). In agrypnia excitata, the circadian rhythmicity is lost and motor activity is increased throughout the $24 \mathrm{~h}$ without any circadian pattern. Electrophysiological recordings show an extreme disorganized pattern with reduced sleep time, brief fragments of alpha-theta activity without sleep elements (probably representing wakefulness), reduction or absence of $\mathrm{K}$ complexes and spindles, loss or small amounts of N3 stage, and brief intrusions or clusters of REM sleep bursts without muscle atonia. Rapid shifts between subwakefulness, NREM sleep features, and REM sleep without atonia are typical during $24 \mathrm{~h}$ recordings [87-91].

Paraneoplastic cerebellar degeneration. Paraneoplastic cerebellar degeneration is characterized by rapid progressive pancerebellar syndrome (trunk and limb ataxia, dysarthria, nystagmus) owing to loss of the Purkinje cells in the cerebellum. It is mostly associated with breast and ovary cancers, small cell lung cancer, and Hodgkin 
disease. Brain neuroimaging is usually normal. Onconeuronal antibodies in paraneoplastic cerebellar degeneration are anti-Yo, anti-Ro, anti Gad65, anti-Tr, anti-amphiphysin, anti-Hu, anti-CARP, and anti-GluR1. In an number of cases, antibodies are not found $[63,64]$.

There is a report of two patients with paraneoplastic cerebellar degeneration and RBD [92]. Two women of 43 and 66 years of age with breast cancer and paraneoplastic cerebellar degeneration (where no onconeuronal antibodies were found) presented with polysomnographic confirmation of RBD. Immunotherapy improved RBD symptomatology but not the cerebellar syndrome. The association of a cerebellar syndrome with RBD suggests that the cerebellum may be implicated in the pathogenesis of this parasomnia. In fact, other disorders where the cerebellum is damaged such as multiple system atrophy [93] and some spinocerebellar ataxias [94] are linked to RBD.

Anti-NMDA receptor encephalitis. Patients typically present with hallucinations, delusions, seizures, short-term memory loss, movement disorders, oculogyric crises, and decreased level of consciousness. It is often associated with young women with ovarian teratomas. Brain magnetic resonance imaging is normal or shows abnormalities in the mesial temporal lobes, basal ganglia and brainstem. Autopsies reveal extensive gliosis, rare T-cell infiltrates and neuronal degeneration in the hippocampus and other regions including the brainstem. Patients can recover after tumor removal and immunotherapy $[63,95,96]$.

The most common sleep abnormality in anti-NMDA receptor encephalitis is insomnia during the acute phase, usually in combination with psychosis [96]. Inversion of sleep pattern with insomnia at night and daytime somnolence occur in $27 \%$ of patients with anti-NMDA receptor encephalitis after recovery [95].

Central hypoventilation is a common finding in young women with ovarian teratoma and anti-NMDA receptor encephalitis. In a recent series of 100 patients with anti-NMDA receptor encephalitis, central hypoventilation occurred in 66 [95]. Central hypoventilation is usually severe requiring mechanical support for a few weeks or many months. Of interest, knockout animals of NR1 subunit of the NDA receptor die of hypoventilation [97].

It is not clear if RBD accompanies anti-NMDA receptor encephalitis. It has been speculated that the movement disorders seen in this paraneoplastic encephalitis (complex bilateral antigravity stereotyped movements of the arms, with perioral and eye movements and less frequently involvement of the legs) represents status dissociatus, but this has never been proved by polysomnography [98]. A case report described a 58-year-old man with anti-NMDA receptor encephalitis who presented with a four month symptomatology somewhat resembling dementia with Lewy bodies (memory loss, aggressive behavior, visual hallucinations). His wife reported acting out violent dreams, vocalizations, kicking, biting, and screaming during sleep occurring one or two times every night. Polysomnography could not be performed to detect RBD or to exclude one of its mimics (e.g., confusional awakenings, severe obstructive sleep apnea) [99].

\section{FUTURE RESEARCH DIRECTIONS}

The study of sleep symptomatology and sleep disorders in the setting of neurological autoimmune diseases opens a window to examine the link between the autoimmune system and the sleep regulation at the molecular, cellular, and anatomic levels. Future research directions include (1) the development of animal models of autoimmune diseases to study the impact on sleep, (2) the identification of the autoimmune basis underlying narcolepsy and anti-lgLON5 disease, (3) the development of adequate and specific immunotherapy to implement in narcolepsy and anti-lgLON5 disease, (3) the adequate management of sleep symptoms in patients with MS, and (4) a new method to score the polysomnographic findings that may occur in patients with antiIgLON5 disease and Guillain-Barré syndrome where conventional rules cannot be applied, and (5) the detailed identification by polysomnography of the sleep disorders that occur in the paraneoplastic syndromes.

\section{FUNDING AND DISCLOSURE}

This review had no funding. The author declares no competing interests.

\section{ADDITIONAL INFORMATION}

Publisher's note: Springer Nature remains neutral with regard to jurisdictional claims in published maps and institutional affiliations.

\section{REFERENCES}

1. American Academy of Sleep Medicine. International classification of sleep disorders, 3rd edn. Darien, IL: American Academy of Sleep Medicine, 2014.

2. Dauvilliers Y, Arnulf I, Mignot E. Narcolepsy with cataplexy. Lancet. 2007;369:499-511.

3. Plazzi G, Pizza F, Palaia V, et al. Complex movement disorders at disease onset in childhood narcolepsy with cataplexy. Brain. 2011;134:3477-89.

4. de Lecea L, Kilduff TS, Peyron C, et al. The hypocretins: hypothalamus-specific peptides with neuroexcitatory activity. Proc Natl Acad Sci USA. 1998;95:322-7.

5. Peyron C, Tighe DK, van den Pol AN, et al. Neurons containing hypocretin (orexin) project to multiple neuronal systems. J Neurosci. 1998;18:9996-10015.

6. Nevarez N, de Lecea L. Recent advances in understanding the roles of hypocretin/orexin in arousal, affect, and motivation. F1000Res. 2018;7:F1000 Faculty Rev-1421.

7. Peyron C, Faraco J, Rogers W, et al. A mutation in a case of early onset narcolepsy and a generalized absence of hypocretin peptides in human narcoleptic brains. Nat Med. 2000;6:991-7.

8. Nishino S, Ripley B, Overeem S, Lammers GJ, Mignot E. Hypocretin (orexin) deficiency in human narcolepsy. Lancet. 2000;355:39-40.

9. Bourgin P, Zeitzer JM, Mignot E. CSF hypocretin-1 assessment in sleep and neurological disorders. Lancet Neurol. 2008;7:649-62.

10. Thannickal TC, Moore RY, Nienhuis R, et al. Reduced number of hypocretin neurons in human narcolepsy. Neuron. 2000;27:469-74.

11. Mahoney CE, Cogswell A, Koralnik IJ, Scammell TE. The neurobiological basis of narcolepsy. Nat Rev Neurosci. 2019;20:83-93.

12. Aran A, Lin L, Nevsimalova S, Plazzi G, Hong SC, Weiner K, et al. Elevated antistreptococcal antibodies in patients with recent narcolepsy onset. Sleep. 2009;32:979-83.

13. Han F, Lin L, Warby SC, Faraco J, Li J, Dong SX, et al. Narcolepsy onset is seasonal and increased following the $2009 \mathrm{H} 1 \mathrm{~N} 1$ pandemic in China. Ann Neurol. 2011;70:410-17.

14. Partinen M, Kornum BR, Plazzi G, Jennum P, Julkunen I, Vaarala O. Narcolepsy as an autoimmune disease: the role of $\mathrm{H} 1 \mathrm{~N} 1$ infection and vaccination. Lancet Neurol. 2014;13:600-13.

15. Latorre $\mathrm{D}$, Kallweit $\mathrm{U}$, Armentani $\mathrm{E}$, et al. T cells in patients with narcolepsy target self-antigens of hypocretin neurons. Nature. 2018;562:63-68.

16. Lecendreux M, Churlaud G, Pitoiset F, et al. Narcolepsy type 1 is associated with a systemic increase and activation of regulatory $t$ cells and with a systemic activation of global T cells. PLoS ONE. 2017;12:e0169836.

17. Pedersen NW, Holm A, Kristensen NP, et al. CD8+ T cells from patients with narcolepsy and healthy controls recognize hypocretin neuron-specific antigens. Nat Commun. 2019;10:837.

18. Abad VC, Guilleminault C. New developments in the management of narcolepsy. Nat Sci Sleep. 2017;9:39-57.

19. Barateau L, Lopez R, Dauvilliers Y. Management of Narcolepsy. Curr Treat Options Neurol. 2016;18:43.

20. Lammers GJ. Drugs used in narcolepsy and other hypersomnias. Sleep Med Clin. 2018;13:183-89.

21. Sabater L, Gaig C, Gelpi E, et al. A novel non-rapid-eye movement and rapid-eyemovement parasomnia with sleep breathing disorder associated with antibodies to IgLON5: a case series, characterisation of the antigen, and post-mortem study. Lancet Neurol. 2014;13:575-86.

22. Högl B, Heidbreder A, Santamaria J, Graus F, Poewe W. IgLON5 autoimmunity and abnormal behaviours during sleep. Lancet. 2015;385:1590. 
23. Simabukuro MM, Sabater L, Adoni T, et al. Sleep disorder, chorea, and dementia associated with IgLON5 antibodies. Neurol Neuroimmunol Neuroinflamm. 2015;2: e136.

24. Montojo MT, Piren V, Benkhadra F, et al. Mimicking progressive supranuclear palsy and causing Tako-Tsubo syndrome: a case report on IgLON5 encephalopathy [abstract]. Mov Disord. 2015;30:710.

25. Brüggemann N, Wandinger KP, Gaig C, et al. Dystonia, lower limb stiffness, and upward gaze palsy in a patient with IgLON5 antibodies. Mov Disord. 2016;31:762-64.

26. Schröder JB, Melzer N, Ruck T, et al. Isolated dysphagia as initial sign of antiIgLON5 syndrome. Neurol Neuroimmunol Neuroinflamm. 2016;4:e302.

27. Haitao $R$, Yingmai $Y, Y a n$, et al. Chorea and parkinsonism associated with autoantibodies to IgLON5 and responsive to immunotherapy. J Neuroimmunol. 2016;300:9-10.

28. Zhang W, Niu N, Cui R. Serial 18F-FDG PET/CT findings in a patient with IgLON5 encephalopathy. Clin Nucl Med. 2016;41:787-88.

29. Gelpi E, Höftberger R, Graus F, et al. Neuropathological criteria of anti-IgLON5related tauopathy. Acta Neuropathol. 2016;132:531-43.

30. Gaig C, Graus F, Compta Y, et al. Clinical manifestations of the anti-IgLON5 disease. Neurology. 2017;88:1736-43.

31. Cagnin A, Mariotto S, Fiorini M, et al. Microglial and neuronal TDP-43 pathology in anti-lgLON5-related taupathy. J Clin Alzheimer's Dis. 2017;59:13-20.

32. Honorat JA, Lomorowski L, Josephs KA, et al. IgLON5 antibody. Neurological accompaniments and outcomes in 20 patients. Neurol Neuroimmunol Neuroinflamm. 2017;4:e385.

33. Bahtz R, Teegen B, Borowski K, et al. Autonatibodies against IgLON5: two new cases. J Neuroimmunol. 2014;275:8.

34. Bonello $M$, Jacob $A$, Ellul MA, et al. IgLON5 disease responsive to immunotherapy. Neurol Neuroimmunol Neuroinflamm. 2017;4:e383.

35. Wenninger S. Expanding the cinical spectrum of IgLON5-syndrome. J Neuromuscul Dis. 2017:4:337-39.

36. Montagna M, Amir R, De Volder I, Lammens M, Huyskens J, Willekens B. IgLON5associated encephalitis with atypical brain magnetic resonance imaging and cerebrospinal fluid changes. Front Neurol 2018;9:329.

37. Tao QQ, Wei Q, Song SJ, Yin XZ. Motor neuron disease-like phenotype associated with anti-IgLON5 disease. CNS Neurosci Ther. 2018;24:1305-08.

38. Ramanan VK, Crum BA, McKeon A. Subacute encephalitis with recovery in IgLON5 autoimmunity. Neurol Neuroimmunol Neuroinflamm. 2018;5:e485.

39. Moreno-Estébanez A, Garcia-Ormaechea M, Tijero B, et al. Anti-IgLON5 disease responsive to immunotherapy: a case report with an abnormal MRI. Mov Disord Clin Pr. 2018;5:653-56.

40. Morales-Briceño $\mathrm{H}$, Cruse $\mathrm{B}$, Fois AF, et al. IgLON5-mediated neurodegeneration is a differential diagnosis of CNS Whipple disease. Neurology. 2018;90:1113-15.

41. Schöberl F, Levin J, Remi J, et al. IgLON5: a case with predominant cerebellar tau deposits and leptomeningeal inflammation. Neurology. 2018;91:180-82.

42. Vetter $E$, Olmes DG, Linker $R$, Seifert F. Teaching video neurolmages: facial myokymia and myorhythmia in anti-lgLON5 disease: The bitten lip. Neurology. 2018;91:e1659.

43. Logmin K, Moldovan AS, Elben S, Schnitzler A, Groiss SJ Intravenous immunoglobulins as first-line therapy for IgLON5 encephalopathy. J Neurol 2019. https://doi.org/10.1007/s00415-019-09221-3.

44. Gaig C, Compta Y Neurological profiles beyond the sleep disorder in patients with anti-IgLON5 disease. Curr Opin Neurol 2019. https://doi.org/10.1097/ WCO.0000000000000677.

45. Hasselbacher K, Steffen A, Wandinger KP, Brüggemann N. IgLON5 antibodies are infrequent in patients with isolated sleep apnea. Eur J Neurol. 2018;25:e46-e47. https://doi.org/10.1111/ene.13566.

46. Mangesius S, Sprenger F, Höftberger R, Seppi K, Reindl M, Poewe W. IgLON5 autoimmunity tested negative in patients with progressive supranuclear palsy and corticobasal syndrome. Park Relat Disord. 2017;38:102-03.

47. Heidbreder A, Philipp K. Anti-IgLON 5 Disease. Curr Treat Options Neurol. 2018;20:29

48. Sabater L, Planagumà J, Dalmau J, Graus F. Cellular investigations with human antibodies associated with the anti-IgLON5 syndrome. J Neuroinflamm. 2016;13:226. https://doi.org/10.1186/s12974-016-0689-1.

49. Dauvilliers $Y$, Mayer $G$, Lecendreux $M$, et al. Kleine-Levin syndrome: an autoimmune hypothesis based on clinical and genetic analyses. Neurology. 2002;59:1739-45.

50. Caminero A, Bartolomé M. Sleep disturbances in multiple sclerosis. J Neurol Sci. 2011;309:86-91.

51. Braley TJ. Overview: a framework for the discussion of sleep in multiple sclerosis. Curr Sleep Med Rep. 2017;3:263-71.

52. Foschi M, Rizzo G, Liguori R, et al. Sleep-related disorders and their relationship with MRI findings in multiple sclerosis. Sleep Med. 2019;56: 90-97.

53. Sakkas GK, Giannaki CD, Karatzaferi C, Manconi M. Sleep abnormalities in multiple sclerosis. Curr Treat Options Neurol. 2019;21:4
54. Gomez-Choco M, Iranzo A, Blanco Y, Graus F, Santamaria J, Saiz A. Prevalence of restless legs syndrome and REM sleep behavior disorder in. Mult Scler Mult Scler. 2007;13:805-08.

55. Plazzi G, Montagna P, Remitting REM. sleep behaviour disorder as the initial sign of multiple sclerosis. Sleep Med. 2002;3:437-39.

56. Enriquez-Marulanda A, Quintana-Peña, Takeuchi Y, Quiñones J. Rapid eye movement sleep behavior disorder as the first manifestation of multiple sclerosis. A case report and literature review. Int J MS Care. 2018;20:180-84.

57. Tippmann-Peikert M, Boeve BF, Keegan BM. REM sleep behavior disorder initiated by acute brainstem multiple sclerosis. Neurology. 2006;66:1277-79.

58. Nishino S, Kanbayashi T, Fujiki N, et al. CSF hypocretin in Guillain-Barré syndrome and other inflammatory neuropathies. Neurology. 2003;61:823-25.

59. Karkare K, Sinha S, Taly AB, Rao S. Prevalence and profile of sleep disturbances in Guillain-Barre Syndrome: a prospective questionnaire-based study during 10 days of hospitalization. Acta Neurol Scand. 2013;127:116-23.

60. Gao J, Li Y, Sun Y, Hu W, Liu Y, An D, et al. The study of sleep disorder factors in patients with Guillain-Barré syndrome. Int J Neurosci. 2016;126:893-98.

61. Bahnasy WS, El-Heneedy YAE, El-Shamy AM, Badr MY, Amer RA, Ibrahim ISE. Sleep and psychiatric abnormalities in Gullian Barré Syndrome. Egypt J Neurol Psychiatr Neurosurg. 2018;54:5.

62. Cochen V, Arnulf I, Demeret $S$, et al. Vivid dreams, hallucinations, psychosis and REM sleep in Guillain-Barré syndrome. Brain. 2005;128:2535-45.

63. Rosenfeld MR, Dalmau J. Paraneoplastic neurologic syndromes. Neurol Clin. 2018;36:675-85.

64. Höftenberfg R, Rosenfeld M, Dalmau J. Update on neurological paraneoplastic syndromes. Curr Opin Oncol. 2015;27:489-95.

65. Silber MH. Autoimmune sleep disorders. Handb Clin Neurol. 2016;133:317-26.

66. Dalmau J, Graus F, Villarejo A, et al. Clinical analysis of anti-Ma2-associated encephalitis. Brain. 2004;127:1831-44.

67. Blumenthal DT, Salzman KL, Digre KB, Jensen RL, Dunson WA, Dalmau J. Early pathologic findings and long-term improvement in anti-Ma2-associated encephalitis. Neurology. 2006;67:146-49.

68. Overeem S, Dalmau J, Bataller L, et al. HCRT-1 CSF levels in anti-Ma2 associated encephalitis. Neurology. 2004;62:138-40.

69. Hoffmann LA, Jarious $\mathrm{S}$, Pellkofer $\mathrm{HL}$, et al. Anti-Ma and anti-Ta associated paraneoplastic neurological syndromes: 22 newly diagnosed patients and review of previous cases. J Neurol Neurosurg Psychiatry. 2008;79:767-73.

70. Landolfi JC, Nadkarni M. Paraneoplastic limbic encephalitis and possible narcolepsy in a patient with testicular cancer: case study. Neuro-Oncol. 2003;5:214-16.

71. Compta Y, Iranzo A, Santamaria J, Casamitjana R, Graus F. REM sleep behavior disorder and narcolpetic features in anti-Ma2 encephalitis. Sleep. 2007;30:767-69.

72. Rojas-Marcos I, Graus F, Sanz G, Robledo A, Diaz-Espejo C. Hypersomnia as presenting symptom of anti-Ma2-associated encephalitis: case study. Neuro-Oncol. 2007;9:75-77.

73. Sahashi K, Sakai K, Mano K, Hirose G. Anti-Ma2 related paraneoplastic limbic/brain stem encephalitis associated with breast cancer expressing Ma1, Ma2, and Ma3 mRNAs. J Neurol Neurosurg Psychiatry. 2003;74:1332-35.

74. Bennet JL, Galetta SL, Frohman LP, et al. Neuro-opthalamic manifestations of a paraneoplastic syndrome and testicular carcinoma. Neurology. 1999;52:864-67.

75. Waragai M, Chiba A, Uchibori A, Fukushima T, Anno A, Tanaka K. Anti-Ma2 associated paarneoplastic neurological syndrome presenting as encephalitis and progressive muscular atrophy. J Neurol Neurosurg Psychiatry. 2006;77:111-13.

76. Scheid R, Voltz R, Guthke T, Sammler D, von Crammon DY. Neuropsychiatric findings in anti-Ma2-positive paraneoplastic limbic encephalitis. Neurology. 2003:61:1159-60.

77. Sutton I, Winner J, Rowlands D, Dalmau J. Limbic encephalitis and antibodies to Ma2: a paraneoplastic presentation of breast cancer. J Neurol Neurosurg Psychiatry. 2000;69:266-68.

78. Barnett $M$, Prosser J, Sutton I, et al. Paraneoplastic brain stem encephalitis in a woman with anti-Ma2 antibody. J Neurol Neurosurg Psychiatry. 2001;70:222-25.

79. Adams C, McKeon A, Silber MH, Kumar R. Narcolepsy, REM sleep behavior disorder, and supranuclear gaze palsy associated with Ma1 and Ma2 antibodies and tonsillar carcinoma. Arch Neurol. 2011;68:521-24.

80. Dauvilliers Y, Bauer J, Rigau V. Hypothalamic immunopathologyin anti-Maassociated diencephalitis with narcolepsy-cataplexy. JAMA Neurol. 2013;70:1305-10.

81. Kritikou I, Vgontzas AN, Rapp MA, Bixler EO. Anti-Ma1- and Anti-Ma2-associated encephalitis manifesting with rapid eye movement sleep disorder and narcolepsy with cataplexy: a case report. Biol Psychiatry. 2018;83:e39-40.

82. Irani SR, Alexander S, Waters $\mathrm{P}$, et al. Antibodies to Kv1 potassium channelcomplex proteins leucine-rich, glioma inactivated 1 protein and contactinassociated protein-2 in limbic encephalitis, Morvan's syndrome and acquired neuromyotonia. Brain. 2010;133:2734-274.

83. Iranzo A, Graus F, Clover L, et al. Rapid eye movement sleep behavior disorder and potassium channel antibody-associated limbic encephalitis. Ann Neurol. 2006;59:178-82. 
84. Cornellius JR, Pittock SJ, McKeom A, et al. Sleep manifestations of voltage-gated potassium channel complex autoimmunity. Arch Neurol. 2011;68:733-38.

85. Tezer I, Erdener E, Sel CC, Mendikanova L, Sagy S, Topcuoglu M. Daytime polysomnography recording in LIG1-related limbic encephalitis. Arch Neurol. 2012;69:145-46.

86. Leypoldt F, Armangue T, Dalmau J. Autoimmune encephalopathies. Ann N Y Acad Sci. 2015;1338:94-114.

87. Lugaresi E, Provini F. Agrypnia excitata: clinical features and pathophysiological implications. Sleep Med Rev. 2001;5:313-22.

88. Liguori R, Vincent A, Clover L, Avoni P, Plazzi G, Cortelli P, et al. Morvan's Syndrome: peripheral and central nervous system and cardiac involvement with antibodies to voltage-gated potassium channels. Brain. 2001; 124:2417-26.

89. Guaraldi $P$, Calandra-Buonaura G, Terlizzi R, et al. Oneiric stupor: the peculiar behaviour of agrypnia excitata. Sleep Med. 2011;12:S64-67.

90. Provini $P$, Marconi $M$, Amadori $M$, et al. Morvan chorea and agrypnia excitata: when video-polysomnographic recording guides the diagnosis. Sleep Med. 2011;12:1041-43.

91. Antelmi E, Ferri R, Iranzo A, et al. From state dissociation to status dissociatus. Sleep Med Rev. 2016;28:1-13.

92. Vale T, Bizari Fernanes do Prado L, Fernnades Do Prado G, Grazian Povoas Barsittini O, Pedroso JL. Rapid eye movement sleep behavior disorder in paraneoplastic cerebellar degeneration: improvement with immunotherapy. Sleep. 2016;39:117-20.

93. Iranzo A, Santamaria J, Rye DB, et al. Characteristics of idiopathic REM sleep behavior disorder and that associated with MSA and PD. Neurology. 2005;65:247-52.

94. Iranzo A, Muñoz E, Santamaría J, Vilaseca I, Milà M, Tolosa E. REM sleep behavior disorder and vocal cord paralysis in Machado-Joseph disease. Mov Disord. 2003;18:1179-83.

95. Dalmau J, Lancaster E, Martinez-Hernandez E, Rosenfield MR, Balice-Gordon R. Clinical experience and laboratory investigations in patients with anti-NMDAR encephalitis. Lancet Neurol. 2011;10:63-74.

96. Dalmau J, Gleichman AJ, Hughes EG, et al. Anti-NMDA-receptor encephalitis: case series and analysis of the effects of antibodies. Lancet Neurol. 2008;7:1091-98.

97. Forrest D, Yuzaki M, Soares HD, et al. Targeted disruption of the NMDA receptor 1 gene abolishes NMDA response and results in neuronal death. Neuron. 1994;13:325-38.

98. Stamelou M, Plazzi G, Lugaresi E, Edwards MJ, Bathia KP. The distinct movement disorder in anti-NMDA receptor encephalitis may be related to status dissociates: a hypothesis. Mov Disord. 2012;27:1360-63.

99. Coban $\mathrm{A}$, Kücükali $\mathrm{Cl}$, Yalcinkaya $\mathrm{N}$, et al. Evaluation of incidence and clinical features of antibody-associated autoimmune encephalitis mimicking dementia. Behav Neurol. 2014;2014:935379. 